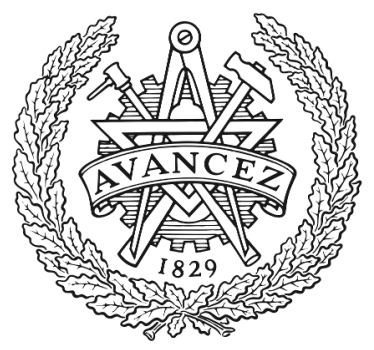

CHALMERS

UNIVERSITY OF TECHNOLOGY

\title{
Sparse-dense MLC for peak power constrained channels
}

Downloaded from: https://research.chalmers.se, 2023-04-26 06:01 UTC

Citation for the original published paper (version of record):

Yoshida, T., Igarashi, K., Karlsson, M. et al (2020). Sparse-dense MLC for peak power constrained channels. European Conference on Optical Communication, ECOC.

http://dx.doi.org/10.1109/ECOC48923.2020.9333148

N.B. When citing this work, cite the original published paper. 


\title{
Sparse-Dense MLC for Peak Power Constrained Channels
}

\author{
Tsuyoshi Yoshida( ${ }^{(1,2)}$, Koji Igarashi(2), Magnus Karlsson(3), and Erik Agrel|((3) \\ (1) IT R\&D Center, Mitsubishi Electric Corporation, Yoshida.Tsuyoshi@ah.MitsubishiElectric.co.jp \\ (2) Graduate School of Engineering, Osaka University \\ (3) Fiber Optic Communications Research Center, Chalmers University of Technology
}

\begin{abstract}
Probabilistic amplitude shaping with Maxwell-Boltzmann distributions can degrade system budgets due to a large peak-to-average power ratio at a low spectral efficiency and at a short reach transmission with few optical amplifiers. We propose a novel coded modulation technique, which is useful in such scenarios.
\end{abstract}

\section{Introduction}

Coherent detection with digital signal processing (DSP) is extending its application range in optical transmission from ultra-long-haul (ULH) to much shorter reach. In $400 \mathrm{~Gb} / \mathrm{s}$ standards of $400 \mathrm{ZR}^{[1]}$ and open ROADM ${ }^{[2]}$, bit-interleaved coded modulation (BICM) with 16-ary quadrature amplitude modulation (QAM) and soft-decision (SD) forward error correction (FEC) codes are utilized; e.g., open FEC (oFEC) ${ }^{[2]}$ based on product codes. For future higher-capacity standards, further advanced coded modulation schemes can be expected.

Reverse concatenation based probabilistic shaping (PS) shows better performance than uniform signaling over optically amplified links ${ }^{[3]}$. Usually the PS is implemented by probabilistic amplitude shaping (PAS) $)^{[4]}$, and the channel can be approximated by a Gaussian channel with an average symbol power constraint. The PAS is powerful for metro to $\mathrm{LH}$, but its advantage is questionable for ULH and short-reach because PAS places nonshaped parity bits to sign bits and constrains base constellation to regular QAMs.

In ULH transmission, we need very high noise tolerance, which is obtained by binary/quaternary phase-shift keying (B/QPSK), 8-QAM, or multidimensional modulation with uniform signaling ${ }^{[5-}$ 8]. On the other hand, to cover such low SEs with PAS, we have to use PS-16-QAM. Then the peak-to-average power ratio (PAPR) becomes significantly larger than uniform signaling, which brings performance degradation due to the nonideal behavior of analog devices and finite precision DSP.

Cost-effective short-reach links have no or few optical amplifiers ${ }^{[1]}$, and a channel model that can be approximated by a Gaussian channel with a peak power constraint. While PS with a MaxwellBoltzmann distribution approaches the capacity of a Gaussian channel with an average power constraint, such a centralized distribution leads to a large amount of optical power loss through the optical modulator, which impedes the loss budget ${ }^{[9]}$. Instead of PS, the optimization of geometric shaping (GS) under a peak power constraint has been studied[10]. Very recently, sparse-dense coded modulation (SpaDCoM) ${ }^{[11]}$ has been proposed for intensity-modulation direct detection systems, which shapes the FEC payload only, whereas the FEC parity is carried by uniform symbols. We can use smaller constellation than 16-QAM for payload symbols by extending SpaDCoM for QAM with coherent detection.

Another important aspect for future standards is the handling of a high system throughput such as $800 \mathrm{~Gb} / \mathrm{s}$ or more. Then the logical processing rate and the corresponding power consumption of SD-FEC can be a bottleneck for hardware implementation. Here multilevel coding $(\mathrm{MLC})^{[12,13]}$ can achieve better performance tradeoff by reducing the throughput of SD-FEC. There is room for further improvement of the MLC scheme ${ }^{[13]}$ to coexist with constellation shaping for low SEs or peak power constrained channels.

Thus, in this work, we propose sparse-dense MLC (SpaD-MLC), which combines sparsedense coding, MLC, and reverse concatenation constellation shaping. We show its usefulness in two scenarios; short reach and ULH applications.

\section{Sparse-dense MLC (SpaD-MLC)}

Fig. 1 shows an exemplified transmitter configuration for the proposed SpaD-MLC. The source information bits are fully shaped to construct an arbitrary base constellation. For example, 9-bits input can be converted into two 25-QAM symbols (here longer block lengths are applicable as shown in scenario\#2 later). Each 25-QAM symbol is indexed with a 5-bit label, which is then converted into two lines of onedimensional (1-D) pulse amplitude modulation (PAM) symbols indexed with 3-bit labels ${ }^{[14]}$. All bit tributaries are shaped because the number of 1$D$ symbol levels can be any natural number. The systematic 1-D MLC FEC encoding is similar to the one used in Ref.[13]. The FEC in this work is oFEC ${ }^{[2]}$ (SD-FEC code rate $R_{\mathrm{sdc}} \approx 0.87$ ) or SD low-density parity check code $\left(R_{\mathrm{sdc}} \approx 4 / 5\right.$, FEC threshold: $5 \mathrm{~dB}$ for QPSK) ${ }^{[15]}$. A low redundancy 


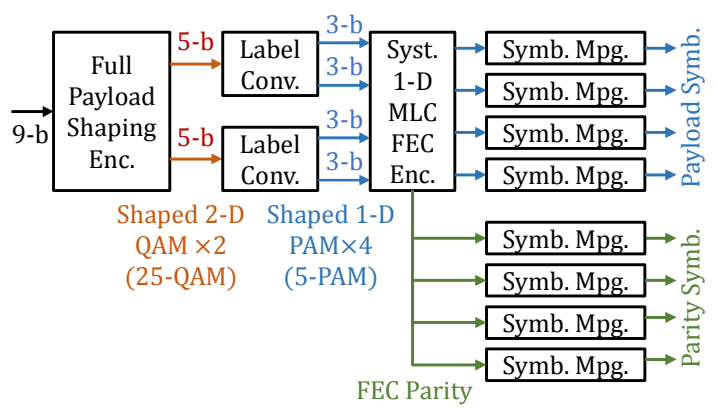

Fig. 1: Exemplified transmitter configuration of SpaD-MLC.

$(\sim 1 \%)$ HD-FEC ${ }^{[16]}$ with a bit-interleaver cleans up the residual errors in cases of SpaD-MLC.

An exemplified frame structure and signalpoint assignment of the proposed method are shown in Fig. 2. An outer HD-FEC protects all bits in the payload symbols, and adds parity bits for the parity symbols. An inner SD-FEC protects LSBs of the payload symbols and the HD-FEC parity bits. The generated SD-FEC parity bits are carried by parity symbols. In this example, we employ three bit tributaries for payload symbols. There are two sets of signal points, i.e., LSB='0' (open markers) and LSB='1' (filled markers) in Fig. 2, which is useful for multistage decoding at the receiver. If needed, the intermediate bit tributary is filled by fixed bits such as '0's. Parity symbols are uniformly distributed Gray-coded PAM symbols. The maximum absolute amplitudes of the payload symbols and parity symbols are $A_{1}$ and $A_{2}$, respectively.

The receiver side processing consists of inverse functions of the transmitter ones. In a systematic MLC FEC decoding, first, LSBs of the payload symbols and parity bits are corrected by SD-FEC decoding. Next, more significant bits are hard-decided based on the SD-FEC-decoded LSBs. A small number of residual errors in payload symbols are corrected by HD-FEC decoding. Decoded labels for 1-D symbols are then converted into the ones for 2-D symbols, and finally recovered to the original source information.

\section{Scenario \#1: Application to short-reach}

First, we apply SpaD-MLC to peak power constrained channels. We examined square 8QAM, 1:1 hybrid of square 8-QAM/16-QAM, 16QAM, 25-QAM, square 32-QAM (the constellation is shown in Fig. 3), 49-QAM, or 64QAM as a base constellation for payload symbols, and uniform 16-QAM for parity symbols. Each payload information rate (IR) is 3 to 6 bit/channel use (bpcu) with a step of 0.5 bpcu.

For benchmarking, we examined three coded modulation schemes. The first is BICM uniform QAMs from 8-QAM to 64-QAM and 1:1 hybrids of neighboring QAMs, where 8-QAM is the star-type

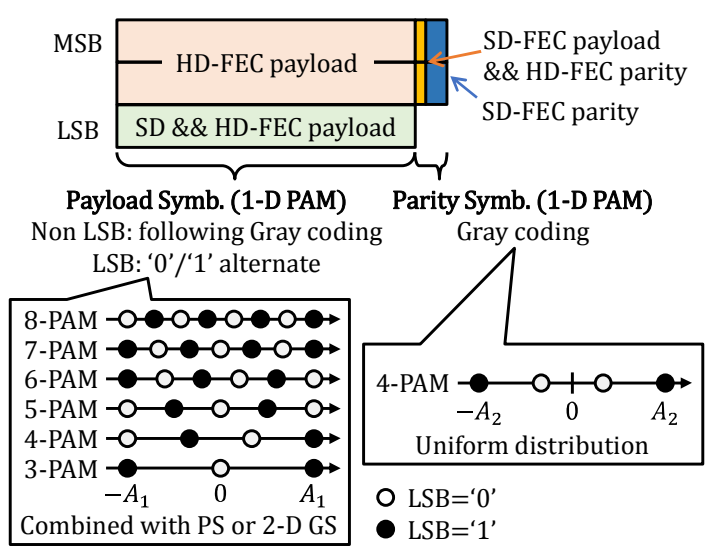

Fig. 2: Exemplified signal format of SpaD-MLC for peak power constrained channels.

and 32-QAM is the cross-type. The second is PSQAM (reducing probabilities of inner PAM levels ${ }^{[17]}$, which is better in this case) with MLC. The third is freezing a portion of LSBs to fixed logic values such as 'O's in MLC, which reduces probabilities of intermediate PAM levels. In terms of the peak SNR ${ }^{[10,14,17]}$, the latter two schemes showed worse performance than BICM (so we will not show the results below in Fig. 3).

We set 1-D peak amplitudes to a constant value in all examined cases even if different constellations were mixed. Though 1-D softdemapping is preferable to simplify the implementation of DSP for short-reach applications, we tried 2-D soft-demapping ${ }^{[18]}$ for square 8-QAM for SpaD-MLC and star 8QAM/cross 32-QAM for BICM QAM.

Fig. 3 shows the theoretically derived required peak signal-to-noise ratio (SNR) over the Gaussian channel at 1 sample/symbol (sps) with a peak power constraint as a function of IR. The peak SNR is defined by PSNR $=\mathrm{SNR} \cdot \mathrm{PAPR}$, where the PAPR is 1-D PAPR at $1 \mathrm{sps}$ computed from the probability mass function of 1-D symbols in this work. In a system without optical amplifiers, the maximum acceptable loss is

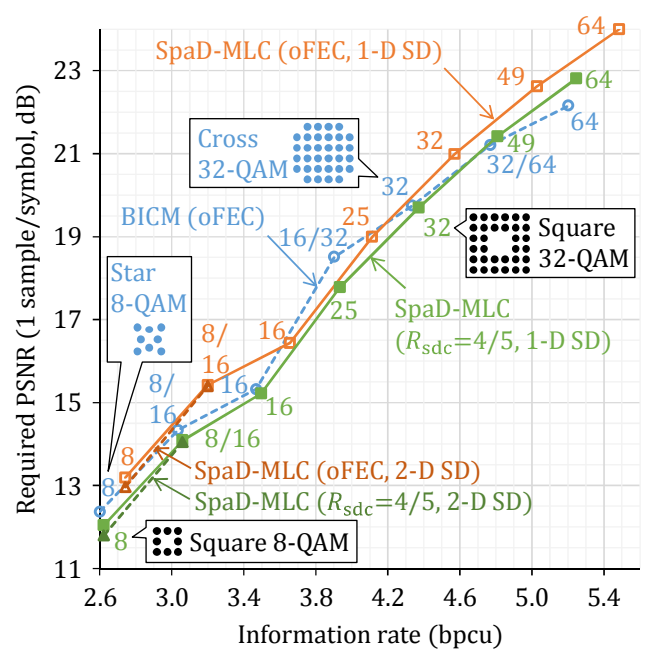

Fig. 3: Required peak SNRs for short-reach applications. 
inversely proportional to the required PSNR. To minimize the required peak SNR, PAM amplitudes were set to $A_{1}=A_{2}$. On the other hand, minimizing the number of signal points by SpaD-MLC showed comparable or better performance except for the regime of IRs less than $4.6 \mathrm{bpcu}$. Especially at $3.9 \mathrm{bpcu}, 0.7 \mathrm{~dB}$ smaller peak SNR (i.e., larger loss budget). Thus SpaD-MLC is useful in peak power constrained short-reach optical channels such as datacenter interconnects. Note that square 32-QAM is also useful for BICM. We observed reduction in required peak SNR by $0.15 \mathrm{~dB}$ from cross 32 QAM.

\section{Scenario \#2: Application to ULH}

Next, we investigate SpaD-MLC as alternatives in low SEs. Fig. 4 shows constellations of SpaDMLC 9-QAM or 5-QAM with QPSK parity. Payload symbols and parity symbols are shown by circles and squares, respectively. Both 9-QAM and 5-QAM are described by two lines of 3PAMs, where each 3-PAM is labelled by 2 bits. In 5-QAM, the outermost signal points are removed from 9-QAM. A 45-degree rotation reduces the 1D PAPR by half.

Fig. 5 shows 1-D PAPR and average symbol power $P_{\text {avg }}$ at a minimum Euclidean distance of 2 at $1 \mathrm{sps}$ as a function of IR. Hierarchical distribution matching ${ }^{[19]}$ was employed to shape SpaD-MLC 9-QAM or 5-QAM symbols with a block length $N_{\mathrm{s}}$ of 192 or 2881 -D symbols. The PAPR of SpaD-MLC 9-QAM and 5-QAM are 4/9 and $2 / 9$ compared with BICM PS-16-QAM $\left(N_{\mathrm{S}}=\right.$ 512 1-D symbols) due to different peak symbol power $P_{\text {peak }}$, while the average symbol powers are almost the same at a given IR by PS.

Then we compared required optical SNR (ROSNR) performance with transceiver noise ${ }^{[20]}$ theoretically at $1 \mathrm{sps}$ with Gaussian noise, whose variance was assumed to be $\sigma^{2}=\sigma_{\mathrm{OSNR}}^{2}+\sigma_{\mathrm{TRx}}^{2}$. The first term corresponds to noise from optical amplifiers at a noise bandwidth equal to the symbol rate. The second term is the transceiver noise. Inside the transceiver, the digital and electrical signals are peak power constrained, and the noise variance can be fixed, so we simply assumed $\sigma_{\mathrm{TRx}}^{2}=k \cdot$ PAPR. Fig. 6 shows a gap between ROSNR and the Shannon limit SNR $\left(2^{\mathrm{IR}}-1\right)$ for various IRs at a coefficient $k$ of $-\infty$, $26,-23$, or $-20 \mathrm{~dB}$. SpaD-MLC 9/5-QAM show better performance than BICM PS-16-QAM in this low SE regime because of their smaller PAPR. For example, the SNR penalty at $k$ of -23 $\mathrm{dB}$ for BICM PS-16-QAM reaches $1 \mathrm{~dB}$, but that for SpaD-MLC 9/5-QAM are less than $0.3 \mathrm{~dB}$, which leads to smaller ROSNR by $>0.5 \mathrm{~dB}$.

\section{Conclusions}

O Payload (2-D LSBs='00’)

O Payload (2-D LSBs $=$ '01' or ' 10 ') $\square$ QPSK Parity

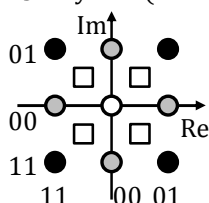

9-QAM Payload (3-PAM $\times 2)$

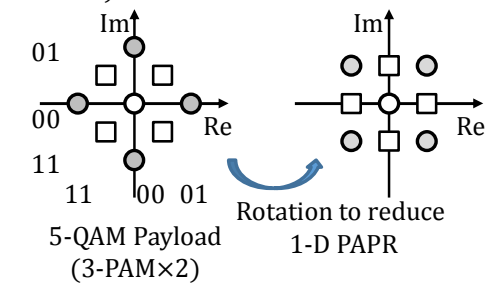

Fig. 4: Exemplified signal points of SpaD-MLC 9-QAM and 5-QAM with QPSK parity for low-SE cases.

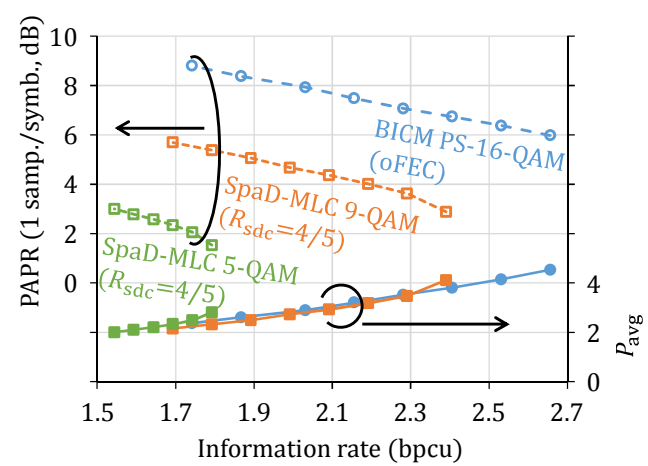

Fig. 5: PAPR and average symbol power $P_{\text {avg }}$.

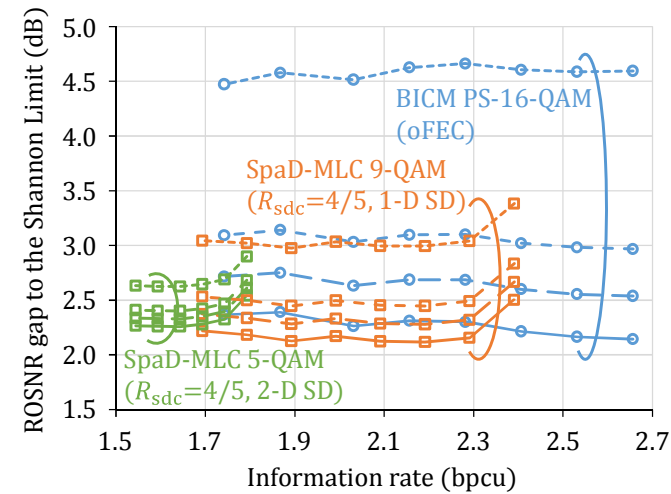

Fig. 6: ROSNR gap with a transceiver noise at $k$ of $-\infty \mathrm{dB}$ (solid), $-26 \mathrm{~dB}$ (long dashed), $-23 \mathrm{~dB}$ (dashed), and $-20 \mathrm{~dB}$ (dotted).

We proposed SpaD-MLC to simultaneously reduce the PAPR and the SD-FEC processing rate. Compared with PAS, SpaD-MLC realizes better performance under channels with (at least partial) peak power constraints, e.g., $0.7 \mathrm{~dB}$ larger loss budget at 3.9 bpcu for short-reach and $0.5 \mathrm{~dB}$ smaller ROSNR at $1.7 \mathrm{bpcu}$ for ULH with a transceiver noise coefficient of $-23 \mathrm{~dB}$.

\section{Acknowledgements}

This work was partly supported by "Massively Parallel and Sliced Optical Network," the Commissioned Research of National Institute of Information and Communications Technology and "The research and development of innovative optical network technology as a new social infrastructure" of the Ministry of Internal Affairs and Communications, Japan. We thank researchers at NTT and Fujitsu for fruitful discussions. We also thank Prof. Kyo Inoue of Osaka University for assistance in the research. 


\section{References}

[1] OIF, "400ZR", [Online]. Available: www.oiforum.com/technical-work/hot-topics/400zr-2/

[2] Open ROADM MSA, [Online]. Available: www.openroadm.org/home.html

[3] F. Buchali, F. Steiner, G. Böcherer, L. Schmalen, P. Schulte, and W. Idler, "Rate adaptation and reach increase by probabilistically shaped 64-QAM: An experimental demonstration", J. Lightw. Technol., vol. 34, no. 7, pp. 1599-1609, Apr. 2016.

[4] G. Böcherer, F. Steiner, and P. Schulte, "Bandwidth efficient and rate-matched low-density parity-check coded modulation", IEEE Trans. Commun., vol. 63, no. 12, pp. 4651-4665, Dec. 2015.

[5] E. Agrell and M. Karlsson, "Power-efficient modulation formats in coherent transmission systems", J. Lightw. Technol., vol. 27, no. 22, pp. 5115-5126, Nov. 2009.

[6] A. D. Shiner, M. Reimer, A. Borowiec, S. O. Gharan, J. Gaudette, P. Mehta, D. Charlton, K. Roberts, and M. O'Sullivan, Opt. Express, vol. 22, no. 17, pp. 2036620374, Aug. 2014.

[7] K. Kojima, T. Yoshida, T. Koike-Akino, D. S. Millar, K. Parsons, M. Pajovic, and V. Arlunno, "Nonlinearitytolerant four-dimensional 2A8PSK family for 5-7 bits/symbol spectral efficiency", J. Lightw. Technol., vol. 35, no. 8, pp. 1383-1391, Apr. 2017.

[8] C. Bin, C. Okonkwo, H. Hafermann, and A. Alvarado, "Polarization-ring-switching for nonlinearity-tolerant geometrically shaped four-dimensional formats maximizing generalized mutual information", J. Lightw. Technol., vol. 37, no. 14, pp. 3579-3591, July 2019.

[9] T. Eriksson, M. Chagnon, F. Buchali, K Schuh, S ten Brink, and L. Schmalen, "56 Gbaud probabilistically shaped PAM8 for data center interconnects", in Proc. Eur. Conf. Opt. Commun. (ECOC), Gothenburg, Sweden, Sep. 2017, Paper Tu.2.D.4

[10] M. Schaedler, S. Calabrò, F. Pittalà, G. Böcherer, M. Kuschnerov, C. Bluemm, and S. Pachnicke, "Neural network assisted geometric shaping for $800 \mathrm{Gbit} / \mathrm{s}$ and 1Tbit/s optical transmission", in Proc. Opt. Fib. Commun. Conf. (OFC), San Diego, CA, USA, Mar. 2020, Paper M1G.1.

[11] A. Elzanaty and M.-S. Alouini, "Adaptive coded modulation for IM/DD free-space optical backhauling: A probabilistic shaping approach", IEEE Trans. Commun., DOI: 10.1109/TCOMM.2020.3006575, July 2020.

[12] K. Sugitani, Y. Koganei, T. Oyama, and H. Nakashima, "Partial multilevel coding with probabilistic shaping for low-power optical transmission", in Proc. OptoElectron. Commun. Conf. and Int. Conf. Photonics in Switching and Computing (OECC/PSC), July 2019, Paper TuB1-5.

[13] T. Yoshida, M. Karlsson, and E. Agrell, "Multilevel coding with flexible probabilistic shaping for rateadaptive and low-power optical communications", in Proc. Opt. Fib. Commun. Conf. (OFC), San Diego, CA, USA, Mar. 2020, Paper M3J.7.

[14] G. Böcherer, "Labeling non-square QAM constellations for one-dimensional bit-metric decoding", IEEE Commun. Lett., vol. 18, no. 9, pp. 1515-1518, Sep. 2014

[15] K. Sugihara, Y. Miyata, T. Sugihara, K. Kubo, H. Yoshida, W. Matsumoto, and T. Mizuochi, "A spatiallycoupled type LDPC code with an NCG of $12 \mathrm{~dB}$ for optical transmission beyond $100 \mathrm{~Gb} / \mathrm{s}$ ", in Proc. Opt.
Fib. Commun. Conf. (OFC), Anaheim, CA, USA, Mar. 2013, Paper OM2B.4.

[16] D. S. Millar, R. Maher, D. Lavery, T. Koike-Akino, M. Pajovic, A. Alvarado, M. Paskov, K. Kojima, K. Parsons, B C.Thomsen, S. Savory, and P. Bayvel, "Detection of a $1 \mathrm{~Tb} / \mathrm{s}$ superchannel with a single coherent receiver", in Proc. Eur. Conf. Opt. Commun. (ECOC), Valencia, Spain, Sep. 2015, Paper Mo.3.3.1.

[17] T. Wiegart, F. Da Ros, M. P. Yankov, F. Steiner, S. Gaiarin, and R. D. Wesel, "Probabilistically shaped 4PAM for short-reach IM/DD links with a peak power constraint", [Online]. Available: www. arxiv.org/abs/ 2005. 12428

[18] T. Yoshida, K. Matsuda, K. Kojima, H. Miura, K. Dohi, M. Pajovic, T. Koike-Akino, D. S. Millar, K. Parsons, and T. Sugihara, "Hardware-efficient precise and flexible soft-demapping for multi-dimensional complementary APSK signals", in Proc. Eur. Conf. Opt. Commun. (ECOC), Düsseldorf, Germany, Sep. 2016, Paper Th.2.P2.SC3.27.

[19] T. Yoshida, M. Karlsson, and E. Agrell, "Hierarchical distribution matching for probabilistically shaped coded modulation", J. Lightw. Technol., vol. 37, no. 6, pp. 1579-1589, Mar. 2019.

[20] L. Galdino, D. Semrau, D. Lavery, G. Saavedra, C. B. Czegledi, E. Agrell, R. I. Killey, and P. Bayvel, "On the limits of digital back-propagation in the presence of transceiver noise", Opt. Express, vol. 25, no. 4, pp. 4564-4578, Feb. 2017. 